DOI: https://doi.org/10.46296/rc.v5i9.0041

\title{
La usabilidad de los medios de Comunicación en la provincia de Santa Elena en tiempo de COVID-19
}

\section{The usability of the media in the province of Santa Elena in the time of COVID-19}

\author{
León-Valle Benjamín Wilson \\ Universidad Estatal Península de Santa Elena. Santa Elena, Ecuador. \\ Correo: bleon@upse.edu.ec \\ ORCID ID: https://orcid.org/0000-0002-3374-5207 \\ Vélez-Bermello Gabriela Lourdes \\ Universidad Estatal Península de Santa Elena. Santa Elena, Ecuador. \\ Correo: gvelez@upse.edu.ec \\ ORCID ID: https://orcid.org/0000-0002-7414-8441 \\ Reyes-Tomalá Brenda Amparito \\ Universidad Estatal Península de Santa Elena. Santa Elena, Ecuador. \\ Correo:breyes@upse.edu.ec \\ ORCID ID: https://orcid.org/0000-0001-6815-8507 \\ Arévalo-Vélez Verónica Lucía \\ Universidad Estatal Península de Santa Elena. Santa Elena, Ecuador. \\ Correo: velu av@hotmail.com \\ ORCID ID: https://orcid.org/0000-0002-8746-7732
}

\begin{abstract}
RESUMEN
La pandemia causada por el COVID-19 ha evidenciado una profunda crisis a todo nivel; social, cultural, tecnológico, político económico, que rebasó la racionalidad del ser humano de forma individual y colectiva, la situación se agravó debido a que los canales de comunicación institucionales perdieron la credibilidad, por la información sesgada, escasa o errónea, lo que dio como consecuencia la aparición de otros actores comunicacionales, asumidos por la ciudadanía que cuenta con dispositivos inteligentes, pasaban información y aunque, quizás, bien intencionada, se desinformaba, ante este panorama crítico, cabe preguntarse: ¿Cuál es el rol que cumplen los medios de comunicación en época de crisis?, y ¿Cuáles fueron con los medios de comunicación más utilizados en la provincia en época de pandemia? En este sentido, determinar la usabilidad de los medios de comunicación en la provincia de Santa Elena en época del COVID-19, constituyó un elemento fundamental, para el establecimiento de mecanismos de comunicación para que sean utilizados con mayor probidad. Metodológicamente se analizaron los estándares de usabilidad de portales Web de de la provincia y luego se profundizó en conocer, bajo una encuesta, cuáles son las formas y medios de comunicación a la que más recurrieron los ciudadanos.
\end{abstract}

Palabras claves: Comunicación, información, noticia, ciudadanía, usabilidad.

Información del manuscrito:

Fecha de recepción: 01 de diciembre de 2021.

Fecha de aceptación: 27 de diciembre de 2021.

Fecha de publicación: 06 de enero de 2022. 


\begin{abstract}
The pandemic caused by COVID-19 has evidenced a profound crisis at all levels; social, cultural, technological, political-economic, which exceeded the rationality of the human being individually and collectively, the situation worsened because the institutional communication channels lost credibility, due to biased, scarce or erroneous information, which gave As a consequence, the appearance of other communicational actors, assumed by citizens who have smart devices, passed on information and although, perhaps, well-intentioned, it was misinformed, faced with this critical panorama, it is worth asking: What is the role played by the media? communication in times of crisis?, and What were the most used means of communication in the province in times of pandemic? In this sense, determine the usability of the media in the province of Santa Elena at the time of COVID-19, use a fundamental element to establish communication mechanisms so that they are used with greater integrity. Methodologically, the usability standards of the province's Web portals were analyzed and then it was deepened to know, under a survey, the results are the forms and means of communication to which the citizens most resorted.
\end{abstract}

Keywords: Communication, information, news, citizenship, usability.

\title{
1. INTRODUCCIÓN
}

La comunicación históricamente ha cumplido con un rol importante en el desarrollo de la humanidad, a través de las herramientas, instrumentos y metodologías apropiadas contribuyen con el análisis, la interpretación, sistematización y publicación de la información. De modo que estos pasos indican un proceso que necesariamente debe cumplirse. Sin embargo, los adelantos tecnológicos en comunicación presentan un verdadero reto por la inmediatez con la que se puede acceder en la actualidad a la información.

Ante la constante evolución de la tecnología, los medios de comunicación han tenido que adaptarse e ir incorporándose a los nuevos cambios y migrar sus contenidos a la plataforma Web, a este fenómeno se lo conoce como la convergencia. El diccionario de la Real Academia de la Lengua Española define a la convergencia como: "el encuentro entre dos puntos, cosas o situaciones que parten de lugares diferentes, para unirse en un punto" (RAE, 2019).

Este proceso de transición no es tan sencillo como parece, implica inversión en infraestructura tecnológica, alojamiento de dominio en la Web, personal especializado, además de condiciones que permitan establecer puentes de comunicación con los ciudadanos. 
Ahora bien, esto conduce a la interacción persona - tecnología, a través de software Booth citado por Fernández la define como: "la interacción o intercambio de signos entre dos o más pares, asignado los participantes en el proceso comunicativo los significados a esos signos" (Fernández, 2018). Ahora bien, se debe destacar que existe una serie de diversidades en la forma de interactuar porque cada persona presenta sus propias necesidades, concepciones, y formas de aprender, por lo tanto, hacer de este proceso algo complejo que debe contemplar algunos principios de interacción. El acceder a una interfaz no debe ser una acción compleja, el éxito depende de su facilidad para su uso.

\section{Definiendo la usabilidad}

Jakob Nielsen (2012) manifiesta que: "La usabilidad es un atributo de calidad que evalúa la factibilidad de usos de las interfaces de usuario. La palabra se refiere también a métodos para mejorar la facilidad de usos durante el proceso de diseño" (Nielsen, 2012). Además, plantea cinco componentes de calidad que determinan la usabilidad de una interfaz; como la capacidad de aprendizaje, la eficiencia, memorabilidad, errores, satisfacción. Es decir, la facilidad que tienen los usuarios para realizar tareas y qué tan agradable es usar el diseño, para relacionarse con la interfaz de un navegador.

La Guía Digital, define a la usabilidad como: "la medida de la calidad de la experiencia que tiene un usuario cuando interactúa con un producto o sistema. Esto se mide a través del estudio de la relación que se produce entre las herramientas y quienes las utilizan" (Guíadigital, 2000). Es decir, la facilidad que tienen los usuarios para ingresar a un dominio de un sitio web. Este término también se usa en el contexto de productos en áreas de conocimiento o trasmiten conocimientos.

De hecho, la usabilidad en los portales Web no es lo único que se analiza, pues la accesibilidad en redes sociales se ha convertido en uno de los principales asuntos a tener presente, Dubois (2012) expresa que "la usabilidad y accesibilidad son características evidentemente fundamentales en cualquier 
aplicación o página web, y las innovaciones en éstas obligan a ir adaptando las metodologías y técnicas de evaluación de la usabilidad" (Dubois, 2012).

Por ejemplo, existen estudios sobre el uso de portales web por parte de personas mayores de edad, por personas con analfabetismo digital y de personas comunes que diariamente ingresan en internet en la búsqueda de información, todos con características diferentes y con cada investigación ciertos portales van mejorando su servicio de accesibilidad, Rodríguez-Palchevich (2012) asegura que para lograr un buen posicionamiento existen estrategias básicas con el fin de que se encuentre más rápida la información (Redríguez , 2012).

En Ecuador existen estudios destinados a la usabilidad Intriago Zambrano (2016) analizó el portal web de elcomercio.com y ecuadorinmediato.com, "La investigación demostró que los usuarios de elcomercio.com se encuentran más satisfechos" (Intriago, 2016), aquello es solo una muestra de la importancia de hacer que el usuario tenga una buena experiencia en su navegación digital.

\section{Importancia de la usabilidad}

La importancia de la usabilidad de un medio de comunicación radica en que es una condición necesaria para la supervivencia, de un sitio web, es decir, si la gente visita, se convierte en un usuario o si abandona no le gusta y se va. En la investigación la importancia es para determinar cuáles son los medios de comunicación con mayor usabilidad en la población santaelenense.

Santa Elena, es una provincia en Ecuador, tiene una población de 308693 habitantes, está ubicada en la zona marino-costera. Según la Agencia de Regulación y Control de las telecomunicaciones (ARCOTEL). 44 medios de comunicación masivos; Radio, Prensa, Televisión, cinco empresas proveedoras de servicio de telefonía e internet.

Durante los meses de marzo, abril y mayo, la pandemia produjo una serie de desinformación, con relación a los hechos que sucedían en el día a día, los medios llamados oficiales, presentaban una cifra que a criterio de otras instituciones y organizaciones de la sociedad civil diferían notablemente. Lo que causaba un clima de inseguridad, tanto física como emocional, a esto se le adiciona, el reporte de los llamados: "periodistas ciudadanos", que con muy 
buena intención informaban a través de las redes sociales los hechos sin ningún tratamiento de la información. La situación se agrava cuando los alcaldes de los gobiernos autónomos descentralizados de la Libertad, Salinas, Santa Elena, indican cifras que difieren con los de las instituciones nacionales, ejemplo en la infografía presentada en marzo, Santa Elena tenía 1 contagiado de COVID-19, mientras que en el territorio se indicaban otras cifras (MSP, 2020).

Ante este escenario caben las siguientes preguntas ¿Cuál es el rol que cumplen los medios de comunicación en época de crisis (COVID - 19) ?, y ¿Qué medios de comunicación fueron los más utilizados en la provincia de Santa Elena en los meses del pico de la pandemia? Y si la usabilidad de los portales web se convirtió en una barrera para que los usuarios accedieran e ingresaran a otras plataformas.

\section{METODOLOGÍA}

Por el enfoque la investigación es mixta, según Hernández (2016) presenta en su libro Metodología de la investigación científica, por el alcance es descriptiva, por la revisión documental, explica que los estudios descriptivos, buscan especificar las propiedades, características, y en el caso de la investigación el perfil de usabilidad de los portales Web de comunicación en la provincia de Santa Elena.

En el proceso investigativo documental, se aplicó la recolección de datos bibliográficos, que consistió en la revisión del objeto de estudio desde las fuentes escritas. Para la recolección de datos desde la fuente primaria, se utilizó la encuesta y la matriz heurística presentada por Nielsen (2012). Instrumento validado en un proceso de investigación aplicado por García (2014) en España. Para el procedimiento y aplicación del instrumento se procedió a aplicar la matriz heurística, además de una encuesta para determinar qué tipo de medio de comunicación en la provincia gozó de mayor sintonía durante los meses críticos de la pandemia. De los seleccionados determinar los criterios de usabilidad. Para el análisis heurístico se observó por 30 días los medios de comunicación de la provincia alojados en la web. La evaluación heurística es un tipo de método de 
inspección de usabilidad donde se verifican si cada elemento de la interfaz de los usuarios concuerda con el principio de usabilidad vigente. Que consiste en obtener los errores de usabilidad de forma rápida, económica y efectiva a través de un cuestionario, que se presenta a continuación.

Matriz No. 1. Matriz heurística.

\begin{tabular}{|l|l|l|l|}
\hline Criterio & Si cumple & Cumple a media & No cumple \\
\hline Tiempo de carga & & & \\
\hline Colocación de logotipo & & & \\
\hline Opción de búsqueda & & & \\
\hline Colocación de la búsqueda & & & \\
\hline Vinculo de Navegación en el pie de la pagina & & & \\
\hline Opción de registro & & & \\
\hline Acerca de la empresa & & & \\
\hline Ayuda & & & \\
\hline Animación & & & \\
\hline Imágenes / ilustraciones & & & \\
\hline Publicidad & & & \\
\hline Color cuerpo de texto & & & \\
\hline Color de vinculo no visitados & & \\
\hline Impresión de la página sin dificultades & & \\
\hline $\begin{array}{l}\text { Los usuarios pueden disfrutar de todos los } \\
\text { contenidos del medio web, sin necesidad de } \\
\text { descargar plugin }\end{array}$ & & & \\
\hline $\begin{array}{l}\text { El sitio web utiliza recursos multimedia } \\
\text { diversos y comprensibles. }\end{array}$ & & & \\
\hline $\begin{array}{l}\text { Dentro del sitio web se puede observar la } \\
\text { utilización de un lenguaje fácil de entender }\end{array}$ & & & \\
\hline $\begin{array}{l}\text { Permite que los usuarios valoren los } \\
\text { contenidos noticiosos. }\end{array}$ & & & \\
\hline $\begin{array}{l}\text { Enlace del medio web con redes sociales a } \\
\text { un solo clic. }\end{array}$ & & & \\
\hline
\end{tabular}

Recuperado de: (García, 2014) http://www.ibersid.eu/ojs/index.php/scire/article/view/1499/1477. Elaborado por: Autores.

Además de la matriz heurística se procedió a la recolección de información a través de una encuesta que fue dirigida a 400 estudiantes de la carrera de Comunicación. Los que constituyen la población a investigar. De la población aplicando la formula muestral, se obtuvo una muestra de 196 estudiantes.

\begin{tabular}{lccc}
$\mathrm{p}$ & $=$ & 0,5 \\
error $\quad=+/-$ & 5 & \\
nivel de confianza & $=$ & 95 & $\%$ \\
Tamaño de la población $=$ & 400 & \\
\multicolumn{2}{r}{ Tamaño de muestra: } & 196
\end{tabular}

La encuesta se la realizó de forma virtual. 


\section{RESULTADOS}

Los resultados obtenidos responden a los medios de comunicación de la provincia que tienen domino web; Antena Tres. Presentando los siguientes datos en cada criterio:

a. Tiempo de carga del medio; se encuentra dentro del rango establecido que es de tres a cinco segundo.

b. Colocación del logotipo; cumple con las características de usabilidad, permite la identificación en el lado superior izquierdo.

c. Opción de búsqueda; es de fácil accesibilidad, permite que el usuario encuentre información de forma inmediata. Además de poseer el link de buscar contenido de interés.

d. Colocación de búsqueda; cumple con los criterios de búsqueda.

e. Vinculo de navegación; presenta vínculos y enlaces para favorecer la navegación y a la búsqueda de información.

f. Color cuerpo de texto; se evidencia legibilidad en el cuerpo del texto.

g. Color de vínculos no visitados; la página web visitada no cumple con este criterio.

h. Color de vínculos visitados; mantiene el mismo color en los vínculos visitados, por lo que se hace un poco complicado identificar este criterio.

i. Impresión de la página sin dificultad; se encuentran alojados en otras plataformas o redes sociales ejemplo Facebook, esto le brinda acceso directamente, está al alcance de los usuarios, ciudadanía en general.

j. Los usuarios pueden disfrutar de todos los contenidos del medio web, sin necesidad de descargar plugin; los usuarios pueden acceder a todo el contenido de la página web.

k. El sitio web utiliza recursos multimedia diversos y comprensibles; Cumple con todos los recursos multimedia. 
I. Dentro del sitio web se puede observar la utilización de un lenguaje fácil de entender; posee un lenguaje sencillo y entendible para todos los usuarios.

m. Permite que los usuarios valoren los contenidos noticiosos. El portal web permite que los usuarios valoren los contenidos, poseen una tabla valorativa. En el que se permite realizar sugerencias.

n. Enlace del medio web con redes sociales a un solo clic. Si cumplen con este criterio.

\section{Resultados de la encuesta}

Los resultados de la encuesta realizada a un segmento de la población universitaria, los revelaron la siguiente situación:

Tabla No. 1. Edad de los encuestados.

\begin{tabular}{cccccc}
\hline & & Frecuencia & Porcentaje & $\begin{array}{c}\text { Porcentaje } \\
\text { válido }\end{array}$ & $\begin{array}{c}\text { Porcentaje } \\
\text { acumulado }\end{array}$ \\
\hline Válido & $18-25$ & 167 & 83,9 & 83,9 & 83,9 \\
& $26-30$ & 20 & 10,1 & 10,1 & 94,0 \\
& $31-35$ & 5 & 2,5 & 2,5 & 96,5 \\
$36-40$ & 4 & 2,0 & 2,0 & 98,5 \\
& $41-45$ & 1 &, 5 &, 5 & 99,0 \\
$46-50$ & 1 &, 5 &, 5 & 99,5 \\
& otros & 1 &, 5 &, 5 & 100,0 \\
\hline Total & 199 & 100,0 & 100,0 & \\
\hline
\end{tabular}

Fuente: Estudiantes universitarios

Elaborado por: Autores.

Con respecto a la pregunta No. 1 , se la realizó con la intencionalidad de determinar la edad de los encuestados, los resultados permiten analizar el segmento que se encuentra muy interesado por los hechos que acontecen en el país. Particularmente en condiciones de crisis. Como dato interesante los resultados indica que el $83,9 \%$ de los encuestados están en un segmento de 18 a 25 años. Lo que indica que existe un segmento de la población relativamente joven que se está interesando por las situaciones y condiciones de la comunidad. Un $10.1 \%$ de 26 a 30 años, que son relativamente joven, un buen indicador de relevo generacional. 
Tabla No. 2. Género de los encuestados.

\begin{tabular}{cccccc}
\hline & & Frecuencia & Porcentaje & $\begin{array}{c}\text { Porcentaje } \\
\text { válido }\end{array}$ & $\begin{array}{c}\text { Porcentaje } \\
\text { acumulado }\end{array}$ \\
\hline Válido & Masculino & 76 & 38,2 & 38,2 & 38,2 \\
& Femenino & 123 & 61,8 & 61,8 & 100,0 \\
\cline { 2 - 6 } & Total & 199 & 100,0 & 100,0 & \\
\hline
\end{tabular}

Fuente: Estudiantes universitarios

Elaborado por: Autores.

Los resultados indican que el $61 \%$ de las personas encuestadas son mujeres, dato que permite inferir la dirección por la que se encuentra estructurando el tejido social, a corto mediano y largo plazo.

Tabla No. 3. En algún momento sitió que la información fue.

\begin{tabular}{cccccc}
\hline & Frecuencia & Porcentaje & $\begin{array}{c}\text { Porcentaje } \\
\text { válido }\end{array}$ & $\begin{array}{c}\text { Porcentaje } \\
\text { acumulado }\end{array}$ \\
\hline \multirow{2}{*}{ Válido } & Excesiva & 73 & 36,7 & 36,7 & 36,7 \\
& Normal & 91 & 45,7 & 45,7 & 82,4 \\
& Mínima & 26 & 13,1 & 13,1 & 95,5 \\
& Muy escasa & 9 & 4,5 & 4,5 & 100,0 \\
\cline { 2 - 6 } & Total & 199 & 100,0 & 100,0 & \\
\hline
\end{tabular}

Fuente: Estudiantes universitarios

Elaborado por: Autores.

Los encuestados manifiestan que la información emitida por los medios de comunicación fue para un $45.7 \%$ normal, es decir, que está dentro de los parámetros de la información, diaria, sin embargo, el 36,7 \% indica que fue excesiva, es un porcentaje medianamente significativo. Con relación a la información recibida el 84 \% indica que fue importante, vs a un 15,1\% que le restó importancia. 
Tabla No. 4. De las siguientes plataformas digitales marque tres en las que usted recibía información de COVID-19 en la provincia.

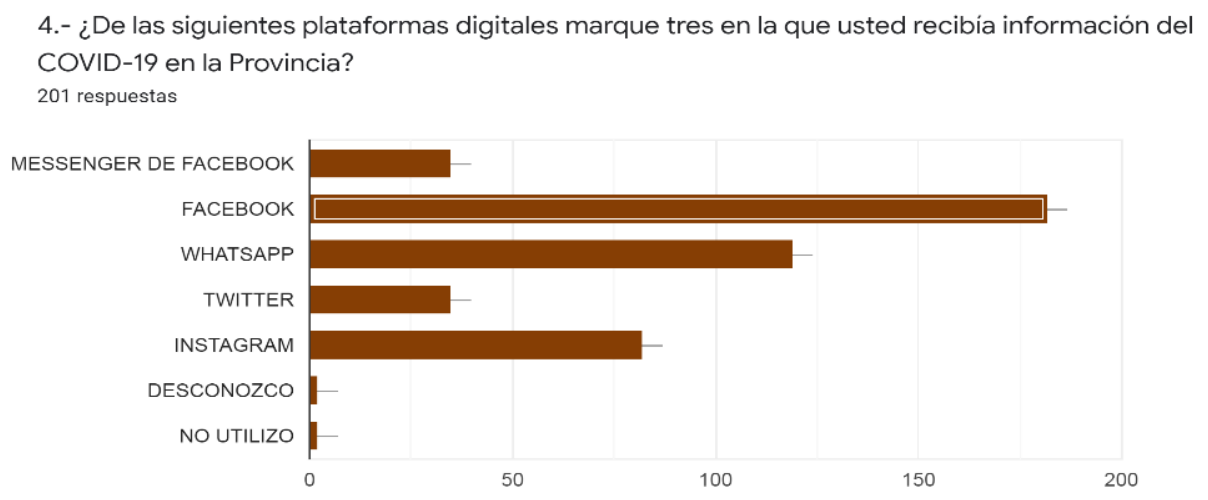

Fuente: Estudiantes universitarios Elaborado por: Autores.

De las plataformas utilizadas los encuestados $90.5 \%$ indican que el Facebook es uno de los medios que más utilizaron para obtener las noticias, luego sigue el WhatsApp con $59.2 \%$, en tercer el Instagram con un $40.8 \%$ lo que indica que las redes sociales se han convertido en uno de los medios de comunicación mas utilizados para informar. Con relación a los medios que utiliza los encuestados manifestaron que el $68 \%$ usa teléfono inteligente, el $52.2 \%$ usa dispositivo portátil y un $22.7 \%$ utiliza PC de escritorio.

Tabla No. 5. De los medios de comunicación tradicionales marque tres en las que usted recibía información de COVID-19 en la provincia

\begin{tabular}{cccccc}
\hline & & & & Porcentaje & Porcentaje \\
Válido & Radio & 29 & 14,6 & 14,6 & 14,6 \\
& Televisión & 139 & 69,8 & 69,8 & 84,4 \\
& Prensa & 16 & 8,0 & 8,0 & 92,5 \\
& Comunicado & 15 & 7,5 & 7,5 & 100,0 \\
\cline { 2 - 5 } & Total & 199 & 100,0 & 100,0 & \\
\hline
\end{tabular}

Fuente: Estudiantes universitarios

Elaborado por: Autores.

Los más medias en época de COVID-19 una vigencia interesante, los encuestados manifiestan que el medio mas utilizado para conocer las noticias es la televisión, con un 69.8 \% de la audiencia. Otro medio de comunicación usado en la provincia de Santa Elena es la Radio, que en otros escenarios tenia un protagonismo importante, sin embargo, se encuentra en un segundo lugar con un $14.6 \%$ de audiencia. Lo que nos permite inferencia que la televisión se ha 
convertido en época de pandemia un medio muy importante para la comunicación.

Finalmente, el $69.3 \%$ de los encuestados indican que utilizan a Google Noticias como buscador de internet, mientras que el $40 \%$ busca noticias en cualquier otro buscador, el $16.8 \%$ no utiliza buscadores, Bing o utilizan un $2.5 \%$ y YAHOO el $1 \%$. Con relación a los buscadores académicos utilizados el $49.5 \%$ no utiliza, el $24.8 \%$ utiliza academia, el $18.8 \%$ utiliza otros que no se encuentran en la lista. El $14.9 \%$ Dialnet. Lo que se puede evidenciar que es que si bien es cierto existe un porcentaje que trata de informarse por medios certificados, un gran porcentaje no los utiliza.

\section{DISCUSIÓN}

La usabilidad como lo expone lo expone Nielsen tiene que ver con la facilidad que tienen los usuarios para acceder a una web, con relación a la encuesta en la pregunta de la tabla No. 4 que los moradores de la provincia de Santa Elena el $90.5 \%$ se informan por Facebook, en la provincia existen algunos medios de comunicación están alojados en esta plataforma, es decir en este sentido se cumple con este criterio de accesibilidad.

Otro aspecto al que se le debe mencionar como resultado de la observación y la búsqueda de la aplicación de la matriz de datos heurísticos propuesta por García, es que los el $80 \%$ de los medios comunicación de la provincia no tienen dominio propio. Como se ha mencionado anteriormente si se encuentra alojados en Facebook. Esto presenta ventaja y desventajas, según se lo ha verificado en la investigación, entre las ventajas, la masificación de la información, mayor cobertura. Entre las desventajas al medio se le resta credibilidad.

\section{CONCLUSIONES}

En Santa Elena históricamente la radio ha sido el medio de comunicación por el que la ciudadanía se informaba, sin embargo, en época de pandemia particularmente, durante el tiempo de observación, los encuestados en el 69. 8 \% manifestaron que utilizaron la televisión como medio de información, esto tiene 
una explicación indirectamente en un criterio de la tabla heurística propuesta por García, que plantea que el uso de imagen es importante para proceder a la usabilidad.

La Radio ocupa el segundo lugar según la encuesta, pero como dato relevante, en la provincia algunas emisoras, entre ellas, se escogió Antena 3, que cumple para medir los criterios de usabilidad propuesto por García. Es importante indicar que estos criterios se establecen para la usabilidad en Web, sin embargo, los principios son generales aplicados a cualquier medio de comunicación alojado en las redes sociales.

La edad de los encuestados es un elemento es el $83.9 \%$, las estadísticas indican que el, estos indican que el $90.5 \% 40 \%$ utilizan Facebook y un $59.2 \%$ uso de WhatsApp., este dato indica que este tipo de plataformas es mucho más accesible y preferible para los jóvenes. Se debe a que el $68 \%$ tiene teléfonos inteligentes $4 \mathrm{G}$. Este tipo de dispositivo permite la navegabilidad y accesibilidad con mayor agilidad.

El criterio de usabilidad que permitan procesos de aprendizaje, según los resultados de la encuesta con relación a los buscadores académicos existe un $49.3 \%$ que no utiliza ninguno, frente a un $24.6 \%$ que utiliza buscadores académicos, estos resultados evidencian que se necesita fomentar una cultura de uso académico, particularmente porque la población realizada la encuesta es una población universitaria.

En conclusión, la investigación muestra la experiencia de los usuarios desde el punto de vista de Nielsen y García, para determinar el criterio de los usuarios para usar uno u otro medio de comunicación. En este sentido los resultados son concluyentes para la provincia de Santa Elena.

\section{REFERENCIAS}

Dubois, J. (2012). Usabilidad en las redes sociales. Obtenido de reunir.unir.net/handle/123456789/51: https://reunir.unir.net/handle/123456789/51

Fernández , P. (2018). elibro.net/es/ereader/upse/106512?as_all=la. Obtenido de elibro.net/es/ereader/upse/: 
https://elibro.net/es/ereader/upse/106512?as_all=la_usabilidad\&as_all_ op=unaccent_icontains\&prev=as

García, J. (2014). Usabilidad de las páginas de inicio de los diarios digitales. Obtenido de http://www.ibersid.eu: http://www.ibersid.eu/ojs/index.php/scire/article/view/1499/1477

Guíadigital. (2000). http://www.guiadigital.gob.cl/articulo/que-es-lausabilidad.html.

Intriago, M. (2016). Usabilidad de periodicos digitales en Ecuador. Obtenido de dspace.udla.edu.ec/handle/33000/5259: http://dspace.udla.edu.ec/handle/33000/5259

MSP. (2020). SITUACIÓN NACIONAL POR COVID-19 (CORONAVIRUS). Obtenido de https://www.salud.gob.ec/wpcontent/uploads/2020/03/INFOGRAFIA-NACIONALCOVI-19: https://www.salud.gob.ec/wp-content/uploads/2020/03/INFOGRAFIANACIONALCOVI-19-COE-NACIONAL-21032020-17H00.pdf

Nielsen , J. (2012). Nilsen Norman Group . Obtenido de nngroup.com/articles/: https://www.nngroup.com/articles/usability-101-introduction-to-usability/

RAE. (2019). Diccionario de la lengua española. Obtenido de dle.rae.es/contenido: https://dle.rae.es/contenido/actualizaci\%C3\%B3n2019

Redríguez , D. (2012). Usabilidad web y posicionamiento en buscadores. Obtenido eprints.rclis.org/16908/1/Eje2_RodriguezPalchevich_44RNB.pdf: http://eprints.rclis.org/16908/1/Eje2_RodriguezPalchevich_44RNB.pdf 